\section{MERCADO Y ACTIVIDAD FÍSICO-DEPORTIVA: LA SALUD Y LA APARIENCIA FÍSICA COMO VALORES DE CAMBIO}

\author{
THE MARKET, SPORTS, AND PHYSICAL ACTIVITY: HEALTH AND PHYSICAL \\ APPEARANCE AS VALUES OF EXCHANGE
}

\author{
MERCADO E ATIVIDADE FISICA ESPORTIVA: A SAÚDE E A APARÊNCIA \\ FÍSICA COMO VALORES DE MUDANÇA
}

Rocío Haydee Arreguín Moreno*, Sergio Alfonso Sandoval Godoy**

\section{Palabras clave} Percepción social. Valores sociales. Ejercicio.

Deportes

\section{Keywords}

Social perception, Social values. Exercise.

Sports.

Palavras-chave

Percepção social. Valores sociais.

Exercício.

Esportes.
Resumen: En este trabajo se analiza la influencia que ejerce el mercado en la actividad físico-deportiva, misma que en la actualidad aparece asociada con una mayor atención sobre el cuerpo. Evidencias documentales y empíricas muestran que a través de la actividad físico-deportiva, el mercado impone, entre otros aspectos, las ventajas de la apariencia legítima dirigidas a fortalecer las relaciones interpersonales y la supremacía del atractivo físico como parámetro de aceptabilidad social y como medida del valor de cambio entre los individuos.

Abstract: In this paper we analyzed the influence of the market in physical activity and sport, which currently appears associated with greater attention to the human body. Document analysis and empirical evidence show that through physical activity and sport, the market asserts the advantages of an ideal/legitimate appearance for strengthening interpersonal relationships and for advancing the supremacy of physical attractiveness both as a parameter of social acceptability and a measure of value of exchange between individuals.

Resumo: Este trabalho analisa a influência que exerce o mercado na atividade física esportiva, mesmo que na atualidade apareça associada como uma atenção destinada ao corpo. Evidências documentadas e empíricas mostram que através da atividade física esportiva o mercado impõe entre outros aspectos, vantagens da aparência legítima dirigida ao fortalecimento das relações interpessoais e supremacia do atrativo físico, como parâmetro de aceitação social e como medida de valor de transformação entre os indivíduos.
* Universidad de Sonora y Centro de Investigación en alimentación y Desarrollo (CIAD, A.C.)

Hermosillo, Sonora, México.

E-mail: rocioarreguin@psicom.uson.mx

${ }^{* *}$ Centro de Investigación en Alimentación y Desarrollo (CIAD, A.C.). Hermosillo, Sonora, México. E-mail: ssandoval@ciad.mx

Recebido em: 30-06-2014 Aprovado em: 12-11-2014 (c) (1) (8) Licence 


\section{INTRODUCCIÓN}

En la actualidad, la preocupación por el cuerpo y la salud se ha vuelto una moda. La mayoría de las personas, especialmente las más jóvenes, sienten una gran presión social por conseguir un cuerpo bello, delgado y joven, ya sea a través de la práctica del ejercicio físico 0 mediante el cuidado de la alimentación, o incluso de ambas. Aunque este ideal no siempre es alcanzable, la motivación por lograrlo ha ido en ascenso, pues se considera una condición de "éxito social". Dicha inquietud por lucir atractivo y saludable tiene un nombre conocido: culto al cuerpo, esto es, lo que algunos autores como Tinning (2002) han llamado somato-centrismo, que indica que el cuerpo se ha convertido en el centro de la vida. De esta manera, el anhelo por el bienestar psíquico y físico a diferencia del pasado, adquiere mayor centralidad lo que genera una constante preocupación por afirmarse ante la mirada de los otros, ansiedad social que, según Valiente (2008), sólo se acalla tras la aceptación, respeto y reconocimiento de los otros.

El interés que tiene el mercado sobre el desarrollo y las transformaciones del cuerpo no es menor, aparece como una sólida relación de influencia ${ }^{1}$ que perfila las características y modalidades de dichos cambios. En esta relación intervienen condicionantes endógenos (como las conductas individuales) y exógenos (como la educación, la política, la salud y los medios de comunicación) que, al parecer, motivan, impulsan o promueven tanto las decisiones de la práctica de la actividad físico-deportiva como la búsqueda de una imagen corporal basada en una "cultura de la delgadez". Todo parece indicar que, forjarse en los valores de cambio del capitalismo moderno se ha convertido socialmente en un deber y en un valor cultural de grandes significados, sobre todo, para las personas que con frecuencia practican el deporte y cultivan el gusto por la apariencia física.

De acuerdo con Inglehart (2008) los valores de cambio cultural que anteriormente se enfocaban en la "dimensión materialista", es decir, en el bienestar material y la seguridad física de las personas, ahora se orientan a la "dimensión posmaterialista" que acentúa más la autoexpresión y realización personal enfocada "al éxito". Por eso, autores como Bajoit (2012, p. 35), señalan que "[...] la sociedad no sólo demanda individuos capaces de disponer de recursos como: alimentación, salud, educación, información, distracción, calificaciones competitivas, empleos creativos y estables y redes de relaciones (de amistad, de amor de sexualidad)", sino también "individuos competitivos, imaginativos, creativos, flexibles, autónomos, responsables y, que además, sean capaces de acumular dinero, como parte de todos los factores con los que será más probable construir la felicidad".

En cierto sentido, lo anterior está ligado a la teoría del cambio cultural (INGLEHART, 2008) que, entre otros aspectos, tal como lo señala Sicilia et al., (2011) "[...] cuando las sociedades adquieren mayores grados de seguridad personal y económica es cuando tienden a abandonar su preocupación por los valores de escasez y supervivencia para orientarse hacia otros valores, como son los de autoexpresión o emancipación". Aunque esto puede ser cuestionable para los países de América Latina y en general para los de menor desarrollo donde los problemas del hambre y la inseguridad pública siguen siendo una constante, existen evidencias que indican que en ciertos estratos de la población, sobre todo de la clase media y alta

1 Empleamos el término influencia en el sentido de Moscovici (1981), como estrategias de convencimiento utilizadas por una minoría o un individuo a través de medios ideológicos y psíquicos para introducir o crear nuevas ideas, nuevos modos de pensar y comportarse, o bien modificar ideas recibidas, actitudes tradicionales y antiguos modos de pensar, todo ello dentro de un entorno en el que no implica igualdad entre las partes. 
(LE BRETON, 2002), tienen cabida este tipo de aspiraciones y valores que enfatizan nuevas orientaciones motivacionales hacia la práctica del ejercicio y la búsqueda de la belleza física. De hecho, son este tipo de valores los que han enfatizado y reforzado las orientaciones somato-centristas de la sociedad actual.

Es por lo anterior que en este trabajo trataremos de probar que a través de estos aspectos, el mercado impone las ventajas de la apariencia legítima en las relaciones interpersonales y sociales, la supremacía del atractivo físico como parámetro de la aceptabilidad social y la exterioridad como medida del valor de cambio.

En lo sucesivo, centraremos el análisis en el mercado, como referente contextual de las prácticas y motivaciones ligadas a la actividad físico-deportiva, cuyos pilares de apoyo son las empresas transnacionales de productos deportivos y de belleza, y quienes a través de los medios de comunicación, instituciones médicas, académicos, dietistas y nutriólogos, entre otros, transmiten lo que bien podría llamarse "el mercado de la buena salud". Asimismo, enfatizamos el papel que desempeñan las presiones sociales de la vida cotidiana, como parte de las conductas individuales y de grupo ligadas a factores de carácter endógeno, donde también se ve reflejada la influencia del mercado. Todo ello con el objetivo de aportar elementos para una mejor comprensión de las actuales transformaciones y tendencias asociadas al cuerpo².

\section{CONSTRUYENDO LA APARIENCIA LEGÍTIMA Y LA ACEPTABILIDAD SOCIAL}

Hoy en día, la asociación entre empresas transnacionales, medios de comunicación e instituciones médicas juegan un papel central en la construcción de una representación social de salud ligada a parámetros de consumo impuestos desde el mercado. Las representaciones del cuerpo saludable y estético que circulan en infinidad de medios de comunicación impresos y electrónicos como el periódico y la revista, los libros y manuales de divulgación, folletos y posters, programas de radio, televisión y cine, además del correo electrónico y sitios web, entre otros, son construcciones sociales con carácter biopolítico que tienen como trasfondo no solo la gestión de la salud sino además, la legitimación social de la apariencia física (PANIER, 2010).

La confluencia de intereses entre estas tres entidades ha ido conformando un sólido engranaje en la formación de la "ortopedia social" cuyo propósito ha sido dirigir a las personas en concordancia con la norma, definiendo aquellas conductas y características consideradas como normales y diferenciándolas de aquellas entendidas como desviadas (FOUCAULT, 1976). La práctica médica se encuentra cruzada por sistemas económicos y de poder que están impactando en las decisiones con respecto al cuidado del cuerpo (cirugía estética, dietas y consumo de aditamentos que combinados con el ejercicio prometen mejores resultados). Es por eso que el mercado de productos "saludables" promovidos por médicos, dietistas y nutriólogos, entre otros, se ha convertido cada vez más en una institución omnipresente y en el soporte ideal para canalizar el poder y producir sujetos consumidores de acuerdo con el interés del capitalismo post-industrial. Todo parece indicar, como acertadamente señala Foucault (1976), que el cuerpo se ha tomado como una realidad biopolítica desde la sociedad industrial, y la medicina como una estrategia que sirve para determinar qué es saludable y qué no lo es.

2 De acuerdo con Turner (1989), en la actualidad el cuerpo deja de ser objeto del discurso sagrado de la carne y pasa a ser objeto del discurso médico, en tanto que se le concibe como "una máquina" que debe ser controlada a través de la ciencia. En este sentido, para este autor -cuya definición representa un supuesto de partida de este trabajo-, el cuerpo es un ente de inspección y vigilancia por parte de las instituciones profesionales, ocupacionales y gubernamentales, cuya regulación es de interés para la salud pública, la economía y el orden político. 
Cabe señalar, sin embargo, que lo que en realidad se percibe en los procesos actuales es la fragilidad de una identidad colectiva y personal, que se ve amenazada por el despliegue de una cultura homogeneizadora y atomizadora que echa mano de los medios de comunicación de masas para imponer la apariencia legítima. No obstante, también se observan resistencias que contrarrestan las fuerzas del mercado y rompen los estereotipos que promueven una estandarización de valores orientados a la estética y a la apariencia. Organizaciones como The National Eating Disorders Association, The Academy for Eating Disorders, The National Association of Anorexia Nervosa and Associated Disorders, se han manifestado en contra de la publicidad que promueve la delgadez extrema (BEHAR, 2010). Comunidades marginadas, indígenas, grupos de inmigrantes, minorías étnicas en todas partes del mundo; y grupos que utilizan técnicas corporales (como la yoga y la danza) con la intención de despertar la consciencia emocional y reflexiva, el conocimiento, la expresión y comprensión de sí mismo (a lo que FOUCAULT, (1990) llamó tecnologías del yo) rechazan los regímenes impuestos donde se demanda el alto rendimiento en el deporte y en el ejercicio físico y diversos programas bajo la intervención política, se niegan a ser asimilados por completo a la cultura del consumismo y replican sus propios valores frente a las normas de la comunidad nacional dominante. En ese sentido la homogenización de una cultura orientada a la estética y cuidado del cuerpo no se puede ligar a un territorio, es más bien una mentalidad que adquiere múltiples particularismos.

\section{DISCURSO DE LA SALUD Y LAS PRÁCTICAS SALUDABLES}

Uno de los argumentos para la promoción de la salud esgrimidos desde la academia, la práctica médica y las instituciones gubernamentales está ligado a la actual epidemia de obesidad que sufre la población de distintos países en el mundo. El egreso económico destinado a enfermedades derivadas del sobrepeso y obesidad gira entre el $2 \%$ y el $9.1 \%$ del gasto total de salud, siendo Estados Unidos el de mayor porcentaje (RODRÍGUEZ; GONZÁLEZ, 2009).

La Organización para la Cooperación y el Desarrollo Económico (OCDE) en su estudio sobre la obesidad y la economía de la prevención (2010), sitúa a México en el primer lugar mundial en índices de sobrepeso y obesidad en adultos. Dicho informe señala que el $70 \%$ de los mexicanos tienen algún grado de sobrepeso y el $30 \%$ sufre de obesidad. Estados Unidos, el país más desarrollado de América del Norte, se ubica en el segundo lugar con $68 \%$ de su población con sobrepeso y $28 \%$ con obesidad. Sin embargo, parece evidente que la verdadera dimensión del problema no es únicamente sanitaria, sino también de naturaleza económica y social. Así, en el mismo informe se indica que los cálculos preliminares del costo de la obesidad y la comorbilidad asociada en países andinos como Bolivia, Colombia y Perú muestran que para la debida atención de la obesidad, tres de sus principales complicaciones clínicas (la diabetes, la hipertensión y la hipercolesterolemia) requerirían recursos financieros por un monto aproximado al 25\% de su presupuesto total destinado a salud. Por ejemplo, entre 1987 y 2002, la proporción de gastos en salud atribuibles a obesidad se incrementó más de 10 veces. En este mismo rubro, según el informe antes citado, México tiene que canalizar 190 mil millones de pesos del gasto público hacia la atención de los problemas de salud asociados a la obesidad, lo que significa la mitad del presupuesto de todas las instituciones públicas de salud (GARCÍA et al., 2010). 
Elevados presupuestos invertidos en la promoción de la actividad física ${ }^{3}$ y en aspectos de salud no han logrado disminuir las enfermedades derivadas de los hábitos sedentarios y de una alimentación inadecuada. Por otra parte, en las últimas décadas se observa, incluso, el aumento de otras enfermedades derivadas de los comportamientos obsesivo-compulsivo, debido a la alta presión social percibida para mantenerse delgado y "saludable", sobre todo, por la población adulta menor a los 30 años (MORENO et al., 2007; THOGERSEN-NTOUMANI; NTOUMANIS, 2006; CAGLAR et al., 2009).

El discurso de la salud, tal como apunta Pedraza (2008), "va encaminado a la autoexploración y al autocontrol como el caudal de autoconocimiento obligado que estimulan las técnicas estético-políticas de movimiento". Estas refieren al mecanismo que promueve en el individuo la capacidad de reconocer el cuerpo y de verse a sí mismo como entidad vulnerable y expuesta y que impulsan estilos de vida saludables con énfasis en los principios de autosensibilidad para el consumo de bienes y servicios. Se brinda un mercado de técnicas, servicios y productos en medio de una oferta excesiva que genera necesidades de conocimiento especializado debido a la amplia variedad de productos saludables existentes. En este proceso de sensibilización y énfasis en la salud dirigido al consumo, se le apuesta al habitus 4 para asegurar la permanencia del mercado de dichos productos (PEDRAZA, 2008).

En ese sentido, el consumo de productos saludables, promete individuos exitosos y fomenta tranquilidad frente al contexto político, económico, social y cultural inestable. Por otra parte, se promueve el consumo asociado con el disfrute, porque es la realización del acto mismo: la fantasía de la plena libertad, pero también en la posesión de determinados artículos, tener para mostrar y obtener el reconocimiento de los otros. Como resulta fácil entender, casi siempre son objetos que se adquieren, pero que no se atesoran porque siempre habrá nuevos y mejorados en detrimento de lo ya adquirido (BOURDIEU, 2006; NUÑEZ, 2010).

Cabe decir que aunque la industria de la dieta promueve un ideal muy delgado, esta difusión no necesariamente conlleva a bajar de peso a largo plazo, sino que dicha moda ha conducido a problemas de salud como la anorexia y la bulimia y otras patologías asociadas con la ansiedad por tener un cuerpo esbelto (VALIENTE, 2008).

Quienes promueven la compra de productos saludables e imponen la norma autoritaria de su consumo no son solamente las transnacionales de los productos de salud, sino también, los profesionales de la salud, principalmente, los médicos dietistas, los nutriólogos, los instructores de ejercicios, los familiares y amigos, y los medios de información, quienes comúnmente recurren a campañas publicitarias con anuncios de personas sonrientes y vigorosas en múltiples espectaculares, revistas y folletos (COUTIÑO, 2012; FINLAY; FAULKNER, 2005). Todo ello se dirige al autocontrol y a la automedicación que ahora invade gran parte de las costumbres de la vida privada, con prácticas nutricionales y de ejercicio que pueden ser en ocasiones inadecuadas. Todo esto, bajo una nueva estrategia biopolítica que apunta desde la mercadotecnia a segmentos y a consumidores específicos con el auge de lo saludable.

3 Para el caso de México, de acuerdo con Ruiz (1996) fue el aparato educativo posrevolucionario el encargado de organizar la práctica físico-deportiva como parte integral de un sistema social que expresa y reproduce los hábitos socioculturales y las formas institucionalmente establecidas para canalizar el tiempo libre, las emociones, las tensiones y expresión corporal del individuo moderno.

4 La noción de habitus se entiende como la interiorización de las reglas sociales, como ley inmanente y orientadora de la acción. Según Bourdieu (2006), se define como un "sistema subjetivo, pero no individual, de estructuras interiorizadas que son esquemas de percepción, de concepción y de acción". Representa una mediación fundamental entre el sistema y el actor, es la forma de articulación del orden social y la práctica de los individuos. En ese sentido, fenómenos culturales como el de la estética y el deporte, entre otros, pueden ser analizados como procesos complejos de producción, distribución y consumo simbólicos. 


\section{LA MERCADOTECNIA DE LA SALUD Y DE LA APARIENCIA}

Sin duda alguna, la práctica del deporte se ha convertido en uno de los más importantes segmentos económicos. Se promueve como una necesidad o demanda de un estilo de vida que se dirige hacia un determinado consumo de bienes y servicios deportivos que, por lo general, son caros y de utilización limitada. Como bien indica Pedráz (2007), todo ello "pasa a ser justificable en nombre de la salud y de la calidad de vida".

Conviene aclarar, sin embargo, la existencia de diferencias significativas entre lo que es la promoción del deporte como espectáculo y como ejercicio físico. El primero es considerado una manifestación estética global, que pone énfasis en atributos de competencia y de estatus social -según sea la práctica deportiva- ${ }^{5}$ para obtener medallas o reconocimiento, ya sea por la adicción al sobreentrenamiento o bien por el consumo de productos debido al fanatismo de algún deporte (asociados con los ídolos o ropa y enseres deportivos) como adaptación al sistema y al comercio, entre otros. El segundo, está más bien asociado a un acto lúdico, al ocio activo, al descanso, a la canalización de la agresividad, al disfrute, a la autoexpresión, a la estética, al equilibrio, a la salud mental -que tiene que ver con la manifestación de sentimientos y sensaciones-, a la transmisión de valores culturales, al autoconocimiento, a la educación, al afianzamiento de la personalidad y al desarrollo de conductas y valores sociales, entre otros (ELIAS; DUNNING, 1992).

En ambos casos, sin embargo, la actividad físico-deportiva se asocia al cuidado del cuerpo debido a que promete alcanzar precisamente la belleza y la salud. Por ello la evaluación física de una persona suele implicar juicios morales y hasta de cordura en términos de personalidad. Así, la gente con sobrepeso puede ser considerada enferma, relajada, desidiosa, indolente y desequilibrada; mientras que a las personas delgadas se les asocia con la salud, la integridad espiritual, la cordura o la sensatez (CONTRERAS; GRACIA, 2005). Estos son algunos de los elementos que toman en cuenta las transnacionales del deporte al momento de lanzar sus campañas publicitarias.

En la actualidad empresas como Nike, ESPN, Adidas, Sky Sports y Under Armour, entre muchas otras, compiten por ocupar importantes nichos de mercado. Sus campañas publicitarias van más allá de promover o impulsar la competencia. Sus elevados niveles de aceptación entre los consumidores de todo el mundo, así como sus altas ventas y ganancias las ubican entre las mejor posicionadas (FORBES, 2014). Por ejemplo, la empresa Nike en 2013 obtuvo ventas globales del orden de los 19 mil millones de dólares (FORBES, 2014); en 2011 las ventas globales (17.3 mil millones de dólares, FORBES, 2011) se repartieron en la siguiente proporción $7.6 \%$ de Norteamérica, $3.8 \%$ de Europa y $2.1 \%$ de países emergentes (SANCHEZ, 2011).

La influencia del mercado de productos deportivos para la práctica de la actividad física, viene asociada a la acción creciente de la industria dietaria, la cirugía estética y los gimnasios. Se ofrecen masajes, fajas y camisetas para reducir talla, tratamientos anticelulitis, cámaras bronceadoras, tonificadoras y/o reductoras, consumo de esteroides anabólicos, e inyecciones de botox, colágeno y ácido hialurónico, entre muchos otros productos. Con ello se trata de imponer una visión hegemónica que se extiende por todos los estratos sociales traspasando límites que antes existían con respecto a la clase social, edad o género, aunque la población de

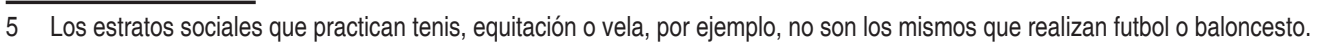


mayor riesgo es sin duda la de adolescentes y la de la mujer 6 . También ha permeado en el ámbito laboral donde se rechaza y se despiden especialmente a las mujeres en función del peso corporal fuera de lo deseado. Se lanzan imágenes publicitarias del prototipo de mujer ideal y control de la dieta, además de que se ejerce en la juventud una fuerte presión para adelgazar, y es mucho el énfasis que se pone sobre las tallas y etiquetas (BARBERO, 2006).

Como consecuencia, la preocupación por el cuidado del cuerpo y la práctica de la actividad físico-deportiva aparece también como un problema de desigualdad y distinción social. La población que tiene menos acceso a las prácticas físico-deportivas, es la formada por la clase social media baja y baja (OMS, 2014). Esta no tiene a su disposición los medios para acudir a los gimnasios y clubs deportivos especializados y culturalmente no pueden adoptar los hábitos de los programas de salud que se tratan de imponer. De tal manera que, cuando los valores y prácticas llegan a ser asumidos por la clase social baja, la clase alta ya los ha transformado lo suficiente como para mantener una distancia social importante. Es entonces que el desarrollo cultural aparece desproporcionado, conservando en esta élite el privilegio de la distinción por el bienestar, mientras que los grupos sociales desfavorecidos siempre parecen llegar demasiado tarde (CONTRERAS; GRACIA, 2005).

\section{CONSUMO CULTURAL Y BELLEZA SIMBÓLICA}

Autores como Bourdieu (2006) y Grogan (2008) argumentan, precisamente, que las diferencias culturales son las principales responsables de los ideales corporales. Lo anterior se debe a que el arte y el consumo cultural han sido creados de forma consciente y deliberada para cumplir una función social de legitimación de las diferencias sociales (BOURDIEU, 2006). Hoy día, la promoción de los prototipos de belleza asociados a la delgadez y a la industria dietaria, juegan también ese papel.

En la construcción de estas diferencias culturales la influencia de la publicidad en medios de comunicación ha sido determinante. Su función ha consistido en manipular los valores culturales existentes para hacer que las empresas transnacionales puedan obtener un mejor posicionamiento en el mercado de los productos que ofrecen. Los anunciantes identifican los profundos anhelos de determinados grupos sociales y bombardean a audiencias incesantemente con promesas de que su vida será buena, feliz y satisfactoria. Como señalan Vanden et al., (2006) los testimonios reflejan frases como "solo quiero la imagen perfecta de muñeca Barbie", "me gustaría tener ese abdomen plano", "me encantaría parecerme a Cindy Crawford", etc. De hecho, la sociedad y los medios de comunicación impulsan a las personas a poner la mirada en alguna imagen que sirva como referente de personas esbeltas y exitosas. En ese sentido, autores como Humberto Eco (2010) indican acertadamente que los medios ponen de nuevo "una iconografía decimonónica, el realismo fabuloso, la exuberancia de Mae West y la gracia anoréxica de las últimas modelos, la belleza negra de Naomi Campbell y la nórdica de Claudia Schiffer, la mujer fatal de tantas transmisiones televisivas o de tantos mensajes publicitarios y la muchacha con la cara recién lavada al estilo de Julia Roberts o de Cameron Díaz" (ECO, 2010). Además de las experiencias de vanguardia se pueden ofrecer modelos de los

\footnotetext{
6 A manera de ejemplo, cabe señalar que un estudio realizado en la ciudad de Hermosillo Sonora, México, evidenció que los productos para adelgazar más consumidos por las mujeres son, en primer lugar, los medicamentos, en segundo lugar los suplementos alimenticios, en tercer lugar las prendas de vestir y calzado deportivo y, por último, los cosméticos. Entre los motivos que el $60 \%$ de las mujeres esgrimieron para consumir productos para adelgazar estaban, primeramente la salud y después la estética. Cabe subrayar que del total de las encuestadas el $77 \%$ indicó que el precio por dichos productos no les parecía caro (COUTIÑO, 2012).
} 
años veinte, treinta, cuarenta o cincuenta, llegando incluso al redescubrimiento de formas ya en desuso.

El consumo cultural, en consecuencia, puede entenderse como una actividad simbólica diferenciada que se aplica no sólo a bienes, artículos y servicios, sino también a otras representaciones sociales, como por ejemplo, la distinción entre ser viejo o joven, o entre ser mujer u hombre. En el primer caso, "pretende describir al 'joven legítimo', una construcción histórica y social, un modelo eminentemente urbano, en cuya imposición influyen con eficacia los medios masivos de comunicación" (BOURDIEU, 2006, p.216). Según el mismo autor uno de los lugares por excelencia al que se acude es el gimnasio, allí se va en busca de reafirmar la juventud, de cuidar el cuerpo y procurar una silueta perfecta. Incluso, las prácticas tan cambiantes como la moda, el uso del tiempo libre, los deportes, las dietas y la música, otorgarán, supuestamente, "legítima juventud" a quienes sigan estos rituales.

En el segundo caso, ciertamente, la feminidad y la masculinidad se encuentran vinculadas con la percepción de que el cuerpo es ante todo una entidad humana. Sin embargo, Cuevas (2009) refiere que, a diferencia del hombre, a la mujer se le oprime y manipula por parte de la cultura global, misma que se construye a partir de la publicidad, los medios de comunicación y la industria cosmética, la cual promueve incluso un estrecho ideal de la belleza femenina occidentalizado a escala mundial.

Cabe señalar, por otra parte, que existen casos "extremos" en que las influencias socioculturales sobre imagen corporal incluyen prácticas de modificación corporal, que incluso llegan a la mutilación y al dolor corporal extremo (cirugía cosmética, piercings, tatuajes y fisicoculturismo) (GONZÁLEZ, 2006). Así, se considera que el consumo es una de las vías principales por la que se canalizan las aspiraciones de lo joven, lo delgado, la búsqueda de la belleza y la salud, asociadas todas con el éxito, y expuestas como promesas fomentadas por el mercado (CUEVAS, 2009).

\section{CONSECUENCIAS ASOCIADAS CON LA PRÁCTICA DE LA ACTIVIDAD FÍSICO- DEPORTIVA, EXCLUSIÓN SOCIAL Y PATOLOGÍAS VINCULADAS AL LOGRO DE METAS PERSONALES}

Al inicio de este escrito señalamos la existencia de factores endógenos que impulsan tanto las decisiones de la práctica de la actividad físico-deportiva como la búsqueda de una imagen corporal basada en una "cultura de la delgadez". Estos tienen que ver con causales psicosociales ligadas a la autodeterminación de los individuos en la búsqueda de sus conceptos de realización personal, en la que frecuentemente aparecen tensiones sociales que explican las conductas individuales y de grupo. En ese sentido, la construcción social del cuerpo a través de sus diferentes manifestaciones, no sólo tiene su explicación en los comportamientos humanos debidos a la influencia del ambiente sociocultural, el mercado y las desigualdades sociales, sino además, a la influencia de factores de conducta autónomos conformados por el autoconcepto y la personalidad.

Siguiendo este parámetro, hay quienes han señalado (GROGAN, 2008; VALIENTE, 2008) que un cuerpo cuidado con esmero simboliza una conducta racional, que representa un alto grado de autoestima y capacidad de autogobierno. Por lo mismo, quien es capaz del dominio del "sí mismo" ejercerá las mismas aptitudes en el plano de las relaciones interper- 
sonales y en el resto de las esferas de la vida. Por lo contrario un físico que no responda a las normas de "alto mantenimiento" identifica a la persona como poco aplicada, perezosa, con escasa predisposición al sacrificio y la responsabilidad. Así, quien no ha cultivado la categoría moral de la autocorrección será más proclive al desorden y la "desviación" que a la lógica de la competencia, la perfección y el éxito.

En el centro de esta "conducta racional" está la ansiedad físico-social, como una consecuencia afectiva que se puede experimentar cuando la gente percibe una respuesta a una evaluación negativa de su físico por parte de los otros (SCOTT, 2004). Se considera como una ansiedad social específica, en la medida que ésta aparece estrechamente vinculada con la imagen y la autoestima corporal y con una importancia destacada en la realización del ejercicio como actividad social, ya que el cuerpo se convierte aquí en el eje central. Así, la ansiedad físico-social parece desarrollarse como un factor influyente relacionado con dichas prácticas. Se manifiesta a través de la preocupación constante por la manera en que el cuerpo es evaluado por otros (HART, et al., 1989). Estas personas regularmente muestran motivación externa hacia la actividad física, en público tienen una mayor conciencia corporal y exhiben perfiles motivacionales similares a los adictos al ejercicio (REEVE, 2003).

Se podría decir que las personas que desarrollan una dependencia al ejercicio tienen características de personalidad basadas en rasgos obsesivos-compulsivos, nerviosismo, extroversión, baja autoestima y altos niveles de ansiedad. De hecho, algunos estudios han destacado la existencia de una asociación negativa con el neuroticismo y la autoestima (HERNÁNDEZ et al., 2005).

Algunas investigaciones que relacionan la ansiedad físico-social (SPA por sus siglas en inglés) con la práctica del deporte han encontrado interesantes resultados. Estudios como los de (MCLACHLAN; HAGGER, 2010), documentaron que los motivos para realizar actividad física de una población del sudeste de Inglaterra estaban asociados con la apariencia y ésta a su vez con motivadores externos, es decir, por el deseo de evitar sentimientos de culpa y vergüenza y para evitar la ansiedad. Los resultados indican que, cuando los motivos por los que esta población realiza actividad físico-deportiva son principalmente para mejorar la imagen corporal, existe menor persistencia y menor sentimiento de bienestar que los que participan por razones de disfrute y autonomía. De hecho, el énfasis en la apariencia explica las altas tasas de deserción en la actividad física.

Asimismo, estudios como el de Grogan (2008) mencionan que en el caso de los hombres atletas, éstos manifiestan desórdenes alimentarios debido a que refieren insatisfacción corporal y a que se enfocan en alcanzar un ideal no realista a través del entrenamiento y la dieta. Para las mujeres también existen diferencias e imposiciones marcadas por la sociedad. En un estudio de caso en Costa Rica se analizó la percepción corporal, la construcción de la femineidad y el consumo de productos de salud y belleza entre jóvenes. Ellas mencionaron que el atractivo físico en relación con ideales estandarizados de belleza "debe ser delgada pero no musculosa", "que se note que haces ejercicio", "cuidadita", entre otras cuestiones. Existen aproximaciones que explican la relación entre la internalización de lo sociocultural y la insatisfacción corporal, y esta última con los desórdenes alimentarios, el neuroticismo, la vigilancia constante del cuerpo y la vergüenza (BAYYARI et al., 2013; LLORENTE et al., 2013).

Asimismo, entre los hombres las implicaciones son igualmente severas. En un estudio de caso realizado en Chiapas, México, mostró que las desviaciones del cuerpo como la 
afección y la enfermedad, son también objetos de evaluación moral. Los participantes dieron testimonio de la percepción de sus propios cuerpos al mencionar algunas prácticas en relación con sus pares, demostraron, por ejemplo, que ser competentes en el deporte, medir su resistencia y fuerza, es necesario para expresar su masculinidad. Con estas acciones mostraron la manera en que desde pequeños los hombres prueban su fuerza física, su fortaleza emocional y el desempeño en los deportes y otras aptitudes para ir adquiriendo el respeto y reconocimiento de los otros (CRUZ, 2006). Así pues, cuando no se dispone de los recursos antes mencionados y no se satisface estos "derechos-deberes", se generan tensiones que afectan el equilibrio en las personas y como consecuencia de esto, enfermedades como la depresión, lo cual a su vez, promueve un nuevo malestar cultural o sentimientos de anomia (BAJOIT, 2012).

\section{CONCLUSIONES}

Los estudios de campo muestran, efectivamente, que las presiones en hombres jóvenes y mujeres para portar un físico ideal son fuerzas sociales que predominan en la sociedad actual. No cumplir con estas normas reales o imaginarias, puede inducir pensamientos y sentimientos negativos hacia la evaluación física de las personas. Cuando esto ocurre se presenta lo que aquí hemos llamado ansiedad físico-social. Como sabemos, ésta lleva a las personas a considerar la idea de participar en actividades físico-deportivas para mejorar su físico y disminuir las posibilidades de evaluaciones negativas, sin embargo, regularmente la ansiedad físico-social socava la motivación hacia la actividad física.

No cabe duda de que las campañas publicitarias y las empresas privadas han creado un entorno que evoca sentimientos de insatisfacción y descontento en los consumidores, al mismo tiempo que provee mensajes que prometen aliviar ese malestar. Como acertadamente señalan Vanden et al., (2006), garantizar que los consumidores tengan una sensación de inadecuación es esencial para la rentabilidad futura de dichas empresas. Por eso, los anuncios promocionales infunden un sentimiento de fracaso, culpa, humillación y vergüenza hacia las personas que no entran en el consumo de "bienes y servicios saludables". Asimismo, atienden a juicios moralistas y emociones que añaden confusión y obstáculos en el desafío de desarrollar estilos de vida saludables (por ejemplo consumir productos y/o servicios de salud de alto costo, como ropa deportiva, aditamentos alimenticios para lograr resultados más rápidos junto con la actividad física, entre otros). Se emiten juicios relacionados con la productividad y el autocontrol, se hace alusión a la pereza y se estigmatiza a quienes no tienen un cuerpo esbelto y/o musculoso o quienes no consumen productos saludables. Por eso, promover sentimientos de inadecuación para "fomentar el odio hacia el cuerpo" se convierte, sin duda, en un negocio muy lucrativo (VANDEN et al., 2006).

Como hemos podido observar hasta aquí, las conductas individuales y motivaciones relacionadas con la actividad físico-deportiva, aunque tienen que ver con la naturaleza del cuerpo, no pueden analizarse al margen del contexto de las transformaciones en la producción y distribución de mercancías dentro de un sistema de consumo masivo, ya que después de todo son consecuencia de los acontecimientos del capitalismo contemporáneo. Por eso hemos tratado de probar que la actividad físico-deportiva y la salud como valores de cambio son transmitidos también por el mercado, mismo que impone las ventajas de la apariencia legítima en las relaciones interpersonales, la supremacía del atractivo físico como parámetro de la aceptabilidad social y del éxito. 
En este sentido, la aspiración fundamental de la sociedad podría estar dirigida a fomentar la construcción de sujetos saludables que, aunque regulados por el mercado, sean capaces de construir su propio mundo simbólico individual y colectivo con potencial para ejercer influencia y forzar cambios en la hegemonía de mercado.

Futuras investigaciones deberán profundizar sobre la asociación entre los motivos de práctica física y la dimensión materialista-postmaterialista para avanzar en el intento por relacionar los motivos del ejercicio físico con las características de las estructuras socio-culturales y económicas de las sociedades occidentales avanzadas. Además tendrán como reto profundizar en los cambios económicos, culturales, políticos y sociales que van sucediendo a través del tiempo y cómo esto afecta a los motivos que la gente tiene para implicarse en sus actividades de ocio, en general, y en la realización de ejercicio físico, en particular.

\section{REFERENCIAS}

BAJOIT, Guy. Las formas contemporáneas de la alienación. En: SUÁREZ, José, H., ZUBILLAGA, Verónica; BAJOIT, Guy (Eds.). El nuevo malestar en la cultura. México: Universidad Nacional Autónoma de México, 2012. p. 35-49.

BARBERO, González José Ignacio. Deporte y cultura: de la modernidad a los tiempos posmodernos. Educación Física y Deporte, Medellín, v. 25, n. 1, p. 25-21, 2006.

BAYYARI, W. D., HENRY, L. J.; JONES, C. Dieting behaviours, obesity and predictors of dieting among female college students at Palestinian universities. Eastern Mediterranean Health Journal, Nasr, v. 19, n. 1, p. 30-36, 2013.

BEHAR, Rosa. La construcción cultural del cuerpo: el paradigma de los trastornos de la conducta alimentaria. Rev Chil Neuro-Psiquiat, Santiago, v. 4, n. 4, p. 319-334, 2010.

BOURDIEU, Pierre. La distinción: criterios y bases sociales del gusto. México: Taurus, 2006. Traducción de Elvira Ruiz, original en francés La distinction [1979].

CAGLAR, Emine; CANLAN, Yusuf; DEMIR, Murat. Recreational exercise motives of adolescents and young adults. Journal of Human Kinetics, Katowice, v. 22, n. 1, p. 83-89, 2009.

CONTRERAS, Jesús; GRACIA, Arnaiz Mabel. Alimentación y cultura: Perspectivas antropológicas. Barcelona: Ariel, 2005.

COUTIÑO, Escamilla Liliana . Consumo de productos para adelgazar en mujeres de 30 a 45 años pertenecientes al estrato socioeconómico medio en Hermosillo, Sonora. Tese (Maestría) - El Colegio de Sonora, Hermosillo, Sonora, 2012.

CRUZ, Sierra Salvador. Cuerpo, masculinidad y jóvenes. Primavera, México D.F., v. 1, n. 1, p. 1-9, 2006.

CUEVAS, Barberousse, Tania. Cuerpo, feminidad y consumo: el caso de jóvenes universitarias.

Revista de Ciencias Sociales, San José, v. 79, n.92, p. 123-124, 2009.

ECO, Humberto. Historia de la belleza. Barcelona: Debolsillo, 2010.

ELÍAS, Norbert; DUNNING, Eric. Deporte y ocio en el proceso de la civilización Fondo de cultura económica, México, 1992. Original en inglés Quest for excitement. Sport and Leisure in the civilizing process [1986].

FINLAY, Sara-Jane; FAULKNER, Guy. Physical activity promotion through the mass media: Inception, production, transmission and consumption. Preventive Medicine, Montreal, n. 40, 121-130, 2005. 
FORBES. Valor de las marcas deportivas según el ranking Forbes, Arte y Sport 2011. Disponible en: http://www.arteysportweb.com/2011/10/04/valor-de-las-marcas-deportivas-segun-el-ranking-forbes/\#. U4pKGnJ5MXY. Acceso en: 1 oct 2012.

FORBES. The Forbes Fab 40: The World's Most Valuable Sports Brands, 2014. Disponible en: http:// www.forbes.com/pictures/mlm45fhklg/1-nike-6/. Acceso en: 20 mayo 2014.

FOUCAULT, Michel. Vigilar y Castigar: Nacimiento de la prisión. Buenos Aires: Siglo XXI Editores Argentina, 1976. Traducción de Garzón del Camino Aurelio. Original en francés Surveiller et punir, [1975].

FOUCAULT, Michel. Tecnologías del yo. Buenos Aires: Paidós, 1996.

GARCÍA-RODRÍGUEZ, José Félix; GARCÍA-FARIÑAS, Anai; RODRÍGUEZ-LEÓN, Gustavo Adolfo; GÁLVEZ-GONZÁLEZ, Ana María. Dimensión económica del sobrepeso y la obesidad como problemas de salud pública. Salud en Tabasco, Tabasco, v. 16, n. 1, p. 891-896, 2010.

GONZÁLEZ, Crussi Francisco. La fábrica en nuestros días. En: La fábrica del cuerpo. México: Ortega y Ortiz, 2006. p. 145-158.

GROGAN, Sarah. Culture and body image. En: Body Image. New York: Routledge, 2008. p. $9-40$

HERNÁNDEZ, Viadel, M.; GUILLEM, Miralles, J. L.; HERNÁNDEZ, Viadel, J. V. Muscle dysmorphia, bigorexia, or the Adonis complex: A case report. Psiquiatria Biologica, Madrid, v.12, n. 3, p.133-135, 2005.

INGLEHART, Ronald. Changing values among western publics from 1970 to 2006. West European Politics, Oxford, v.31, n.1/2, p. 136-146, 2008.

LE BRETON, David. Antropología del cuerpo y modernidad. Antropología del cuerpo y modernidad. Traducción Paula Mahler. Buenos Aires: Ediciones Nueva visión, 2002.

LLORENTE, , E.; WARREN, C. S.; de EULATE, L. P.; GLEAVES, D. H. A Spanish Version of the Sociocultural Attitudes Towards Appearance Questionnaire-3 (SATAQ-3): Translation and Psychometric Evaluation. Journal of Clinical Psychology, Wiley, v. 69, n. 3, p. 240-251, 2013.

MCLACHLAN, Sarah; HAGGER, Martin S. Associations between Motivational Orientations and Chronically Accessible Outcomes in Leisure-Time Physical Activity: Are Appearance-Related Outcomes Controlling in Nature? Research Quarterly for Exercise and Sport, Norwich, v. 81, n. 1, p. 102-107, 2010.

MORENO, Murcia Juan Antonio; LÓPEZ DE SAN ROMÁN, Blanco María; MARTÍNEZ, Galindo Celestina.; ALONSO, Villodre Néstor; GONZÁLEZ-CUTRE, Coll David. Effects of the gender, the age and the practice frequency in the motivation and the enjoyment of the physical exercise. Fitness and Performance Journal, Rio de Janeiro, v. 6, n. 3, p. 140-146, 2007.

MOSCOVICl, Serge. Psicología de las minorías activas. Madrid: Morata, 1981.

NUÑEZ, Noriega Guillermo. "Lo que el desarrollo se llevó" modernidad y nostalgia en Sonora. Estudios Sociales, Hermosillo, n. 17, p. 42-75, 2010.

ORGANIZACIÓN para la Cooperación y el Desarrollo Económico. Obesidad y las economías de la prevención, 2010. Disponible en: http://www.oecd.org/centrodemexico/medios/ obesidadylaeconomiasdelaprevencion.htm. Acceso en: 20 oct. 2014.

ORGANIZACIÓN MUNDIAL DE LA SALUD. Estrategia mundial sobre régimen alimentario, actividad física y salud, 2014. Disponible en: http://www.who.int/dietphysicalactivity/factsheet_ inactivity/es/. Acceso en: 20 oct. 2014.

PANIER, Hernán. La salud como consumo. La construcción del cuerpo saludable como dispositivo biopolítico en la Sociedad de Control. JORNADAS NACIONALES DE INVESTIGADORES 
EN COMUNICACIÓN, 14. 2010. Memorias de las Jornadas Nacionales de Investigadores en Comunicación. Buenos Aires, Argentina, 2010. Disponible en: http://webiigg.sociales.uba.ar/iigg/ jovenes investigadores/5jornadasjovenes/EJE7/Panier - La salud como consumo.pdf. Acceso en: 10 oct. 2012.

PEDRAZ, Miguel Vicente. La construcción de una ética médico-deportiva de sujeción: El cuerpo preso de la vida saludable. Revista de Salud Pública de México, Cuernavaca, v. 49, n. 1, p. 71-78, 2007.

PEDRAZA, Gómez Zandra. De la educación física y el uso de sí: ejercicios estético-políticos de la cultura somática moderna. Movimento, Porto Alegre, v. 14, n. 2, p. 13-37, 2008.

REEVE, Johnmarshall. Motivación y emoción. México: McGraw Hill, 2003.

RODRÍGUEZ, Caro A.; GONZÁLEZ, López B. . El trasfondo económico de las intervenciones sanitarias en la prevención de la obesidad. Rev Esp Salud Pública, Madrid, v. 83, n. 1, p. 25-41, 2009.

RUIZ, Murrieta Rosa María. La estructura social del deporte y su práctica en Sonora 1900-1994: Un ejercicio de periodización histórica. Tese (Maestría) - El Colegio de Sonora, Hermosillo, Mexico, 1996.

SÁNCHEZ, Reyes Cristina. Las marcas deportivas más valiosas del 2011. El economista. México, DF. Disponible en: http://eleconomista.com.mx/deportes/2011/12/12/las-marcas-deportivas-masvaliosas-2011. Acceso en: 20 oct. 2012.

SCOTT, Lisa A.; BURKE Kevin L.; JOYNER Barry A.; BRAND, J. S. Examining the stability of the 7-item social physique anxiety scale using a test-retest method. Measurement in Physical Education and Exercise Science, Philadelphia, v. 8, n. 2, p. 57-62, 2004.

SICILIA, Camacho Álvaro; ÁGUILA, Soto Cornelio.; GONZÁLEZ-CUTRE, David. Valores posmodernos y motivación hacia el ocio y el ejercicio físico en usuarios de centros deportivos. Rev. Int. Cienc. Deporte, Torrelodones, v. 7, n. 5, p. 320-335, 2011.

TINNING, Richard; Glasby Trish. Pedagogical Work and the 'Cult of the Body': Considering the Role of HPE in the Context of the 'New Public Health'. Sport, Education and Society, Waikato, v. 7, n. 2, p. 109-119, 2002.

THOGERSEN-NTOUMANI, Cecilie.; NTOUMANIS, Nikos. The role of self-determined motivation in the understanding of exercise-related behaviors, cognitions and physical self-evaluations. Journal of Sports Sciences, Londres v. 24, n. 4, p. 393-404, 2006.

TURNER, Brian, S. El cuerpo y la sociedad: Exploraciones en teoría social. México, D.F.: Fondo de Cultura Económica, 1989.

VALIENTE, Enrique. Anorexia y bulimia: el corsé de la autodisciplina, En: MARGULIS, Mario (Ed.) La juventud es más que una palabra: Ensayos sobre cultura y juventud, Buenos Aires: Biblos, 2008.

VANDEN, Heede Fred; PELICAN, Suzanne; HOLMES, Betty; MOORE, Sylvia A.; BUCHANAN, David. Values, body weight, and well being: the influence of the protestant ethic and consumerism on physical activity, eating, and body image. Applied Research and Evaluation, Nueva York, v. 25, n. 3, p. 239270, 2006. 
Endereço para correspondência:

Rocío Haydee Arreguín Moreno

Divisaderos 386, colonia Lomas de Madrid - C.P. 83103, Hermosillo, Sonora - México

Financiación: Centro de Investigación en Alimentación y Desarrollo, A.C.; Universidad de Sonora 